http://dx.doi.org/10.12775/szhf.2015.010

Anita Pacholik-Żuromska

Uniwersytet MikoŁaja Kopernika, Toruń

\title{
Literarische Psychoanalyse der Frauengestalten in den gewählten Erzählungen von Arthur Schnitzler
}

\author{
Einführung
}

Es wurde nicht einmal versucht, die Frauennatur zu begreifen und in einen theoretischen Rahmen der Psychologie zu schließen. Es ist im Falle dieses Essays nicht anders. Es wird eine Probe unternommen, Frauenbilder in den gewählten Erzählungen von Arthur Schnitzler: Die Braut, Fräulein Else und Die griechische Tänzerin zu analysieren. Die dargestellten Überlegungen betreffen vor allem den Konflikt zwischen den unterbewussten Urelementen der Frauennatur und ihrer oberflächlichen Existenz, die von Kultur und Gesellschaft determiniert wird. In diesen Erzählungen stößt man sowohl auf ein realistisches Frauenabbild - die Frau ist hier eine Vertreterin der Wiener Gesellschaft des Jahrhundertendes - als auch auf eine Idealisierung bzw. Verallgemeinerung, die versucht, das Ursprüngliche in der Frauenpsyche zu entdecken. Schnitzlers Werke stellen eine wunderbare Psychoanalyse der Protagonistinnen dar. Sie sind eine faszinierende Expedition in die Tiefe der Seele, welche die geheimen menschlichen Wünsche, Lüste und Sehnsüchte entdecken lässt. 
„Wollen Sie mehr über die Weiblichkeit wissen, so befragen sie ihre eigenen Lebenserfahrungen, oder Sie wenden sich an Dichter", sagt Freud. ${ }^{1}$ Bestimmt darf man Schnitzler als einen Forscher der weiblichen Psyche bezeichnen, der die Sprache der Literatur benutzt, um diese beobachteten Phänomene zu beschreiben. Seine Erzählungen berühren die philosophische Dimension der Überlegungen über das Körper-Geist-Problem, wo sich die psychologischen Gründe und körperlichen Determinanten des Verhaltens nicht einfach voneinander trennen lassen. In diesen dualistischen Hinsicht ist der Mensch ein psycho-physische Individuum - ein grundlegendes Element der Gesellschaft und Kultur. Gleichzeitig ist die Kultur ein Käfig, den der Mensch sich erbaut hat. „Jede Kultur zwingt dazu, bestimmte ureigene Triebe einzuschränken. Der Mensch lebt also in seinen Kulturen immer in einem Widerspruch zu seinen genetischen Instinkten."2 Darin sind sowohl Frauen als auch Männer gefangen. Schnitzler entlarvt aber den Geschlechtstrieb hinter der gesellschaftlichen Fassade nicht, sondern führt die Geschlechtlichkeit in den Formen der Prostitution, Tändelei, Liebe, Ehe auf die gesellschaftlichen Bedingungen zurück. ${ }^{3}$

\section{Die Braut}

Besonders stark selbstbewusst ist die Protagonistin der Erzählung Die Braut. Das Werk gehört zu den späten Erzählungen von Arthur Schnitzler. Es wurde zum ersten Mal im Jahre 1932, also schon nach dem Tode des Autors gedruckt. In der kurzen Form überträgt Schnitzler eine ganze Menge an Informationen auf diese Weise, dass man diese Erzählung „lapidar” nennen kann. Der Ich-Erzähler spielt hier eine Doppelrolle. Er erzählt und analysiert gleichzeitig die Situation, an der er auch teilgenommen hat. So ist diese Erzählung eine Reminiszenz des vorherigen Geschehens, die erklären sollen, wie es dazu gekommen ist, dass die Protagonistin die Entscheidung freiwillig

\footnotetext{
${ }^{1}$ S. Freud, Vorlesungen zur Einführung in die Psychoanalyse, Bd. I, 2. Aufl., Frankfurt am Main 1970, S. 565.

${ }^{2}$ Vgl. S. Freud, Das Unbehagen in der Kultur, [in:] Das Unbehagen in der Kultur und andere kulturtheoretische Schriften, Psychologie Fischer, Frankfurt am Main 2001.

${ }^{3}$ Vgl. R. Klüger, Schnitzlers Damen, Weiber, Mädeln, Frauen [Vortrag vom 25. Mai 2000], Picus Verlag, Wien 2001, S. 37.
} 
getroffen hat, eine Liebesdienerin zu werden. Das war keine Notwendigkeit, sie hatte keinen finanziellen oder sozialen Grund dafür.

Sie war aus einer guten Familie, aus einer sehr geachteten und bekannten, behauptete sie sogar, und man hatte sie zu Hause streng erzogen. Aber ihre Sinne erwachten früh und in heftigem Verlangen. In den einsamen Nächten ihrer frühreifen Mädchenzeit hatte sie viele Qualen zu überstehen, und ein seltsamer Vorsatz bildete sich in ihr, aus unklaren Wünschen zu immer festerer Gestaltung. Sie wollte warten, bis sich der Gatte gefunden, denn das musste sie wohl, dann aber, wenn die Gefahr vorüber, wollte sie sich freimütig den ursprünglichen und wilden Trieben ihrer Natur, wollte sich jedem hinschleudern, der ihr gefiel... Männerschönheit und Männerstärke genießen, wo sie sich bot. ${ }^{4}$

Es ist etwas sogar Anstößig in dieser Gefühlbeschreibung. Eine Jungfrau würde eher mit Unschuld und Tugend, aber auf keinen Fall mit den klar ausgebildeten, bewussten sexuellen Neigungen assoziiert werden. Das ist aber sehr frappierend, dass sie sich dessen bewusst ist, was sie fühlt und was sie will, sogar dann, wenn ihre Wünsche als so schockierend erscheinen.

Philosophisch gesehen ist diese Geschichte ein Beispiel des bekannten Problems von Selbstbewusstsein und Willensfreiheit. Die ganze Beschreibung des Prozesses, beginnend damit, wie eine Idee im Kopf entsteht, über ihre Verwirklichung, bis hin zu den Konsequenzen der Entscheidung am Ende, kann zu den Berichten über die Berufung verglichen werden. Die Verkündung, Dirne zu werden, kann selbstverständlich schockieren. Schnitzler hat jedoch eine besondere Einstellung zu der Sexualität, die er wie jede andere Erscheinung in der gesellschaftlichen Realität platzierte. Er spricht wie Freud offen darüber, was die Kultur versteckt. Die Sexualität der Frau, die ein Objekt des männlichen Begehrens ist, gehört zu den natürlichen Trieben, deren sich der Mensch vor allem schämt. Diese Triebe situieren den Menschen im Rahmen der Naturwelt. Sie sind etwas tierisches, materialistisches aber auch natürliches, trotzdem sollen sie verdrängt und durch die Kulturprodukte ersetzt werden. Doch sind diese Triebe stärker als die kulturellen Verbote, was bedeutet, dass sie sich früher oder später in Erscheinung treten.

Der Interpretation, dass die wilden Triebe von der Kultur gefesselt sind, widerspricht die These von Ruth Klüger:

${ }^{4}$ A. Schnitzler, Die Braut, [in:] Gesammelte Werke. Die erzählenden Schriften, 2 Bände, Bd. 1, Frankfurt am Main 1961, S. 83-89. 
[Der Autor] anders als Freud zeigt nicht, dass der Trieb stärker ist als gesellschaftliche Normen und sich diese dienstbar macht, sondern er zeigt, wie jeder und jede in die vorgeschriebenen Fußstapfen steigt auch dort, wo's ums Intimste geht. ${ }^{5}$

Diese Darstellungsweise der Menschen und ihre Sitten oder einfachen Handlungen passierten nicht oft bei Schnitzler. Bestimmt findet dies in Die Braut nicht statt. Ganz im Gegenteil, die Protagonistin steigt über die gesellschaftlichen Normen. Diese Normen sind künstlich von der Definition her. Die Protagonistin verkörpert hingegen das Natürliche und ist mit ihrer Natur einverstanden.

Es ist sehr ungewöhnlich, dass eine Frau freiwillig eine Dirne werden möchte, um eine Glücklichkeit zu finden. Vielleicht deswegen wird Die Braut in den Kategorien des Wahnsinns interpretiert. Diese Art des Wahnsinns, der mit universalen Wahrheiten oder ästhetischen Maßstaben nichts mehr zu schaffen hat, bezeichnen die Kommentatoren von Schnitzler als „Studium des Falls". In diesem Kontext wäre Die Braut das Studium des Wahnsinns. ${ }^{6}$

In Die Braut ist der Körper ebenso wichtig wie der Geist. Wie es Freud in der Psychoanalyse vorausgesetzt hat, sind sie voneinander abhängig und sie beeinflussen sich wechselseitig. Während der Interpretation dieser Erzählung kann man die These aufstellen, dass im Fall der Protagonistin der Körper ihre Psyche determiniert. Das sind die Triebe, denen man nicht widerstehen kann. Der Körper determiniert damit das Schicksal der Protagonistin. Bestimmt wollte die Protagonistin auch etwas mit ihrer Ansicht demonstrieren: „Ihre klugen und ruhigen Augen hatten mir gefallen und das dunkelblaue Kleid, das sie trug. Sie war nicht maskiert und machte durchaus kein Hehl aus ihrer wahren Person." " Dass sie keine Larve trug, sollte vielleicht bedeuten, dass sie nichts zur Verbergen hatte, und noch mehr, dass sie sich enthüllen will.

Das Motiv der Körpermacht wurde schon vor langer Zeit in der Mythologie dargestellt. Es genügt die Zauberin Circe zu erwähnen, die verführerische Frau, die ihre sexuelle Macht benutzt hat, um Odysseus zu betören. Das ist etwas natürliches, den Lüsten zu folgen. Das ist eben eine ungestörte Lebensfreude. Diese Erzählung ist also eine Art der Enttabuisierung des Physischen, dessen sich Menschen oft schämen. Die Scham ist ein untrennbares Element

\footnotetext{
${ }^{5}$ R. Klüger, Schnitzlers Damen, Weiber, Mädeln, Frauen, S. 38.

${ }^{6}$ R. Urbach, Schnitzler-Kommentar zu den erzählenden Schriften und dramatischen Werken, Winkler Verlag, München 1974, S. 40.

${ }^{7}$ A. Schnitzler, Die Braut, S. 86.
} 
des Strebens nach Selbsterhaltung, denn sie ist eine soziale Erscheinung. Man schämt sich vor Gott, vor sich selbst, vor anderen Mitbürgern. Die Scham ist also eine Relation zwischen dem Individuum und den Anderen. Sie gehört nicht zu einer privaten Sphäre, sondern zu den öffentlichen. Nach der Bibel begleitet die Scham den Menschen seit der Erbsünde an. Auf Gottes Frage: „Warum hast du dich vor mir versteckt?”, antwortete Adam: „Ich habe mich geschämt, weil ich nackt bin.” Diese Scham verschwindet aber, wenn der Mensch seine Körperlichkeit akzeptiert. Wenn er damit einverstanden ist, was ihm von Natur aus angehört. Die Protagonistin von Die Braut hat das verstanden. In ihrem Fall hat diese Selbstaffirmation eine extreme Form angenommen.

Der menschliche Geist, der doch ein Individuum charakterisiert, der eben das Wesen der Menschen ausmacht, untersteht hier den Gesetzen des Physischen. Ist das aber nicht eine Verwirklichung dessen, was Bergson „elan vitale" und Heidegger „authentische Existenz" genannt haben? Doch ist das auch eine Realisierung der altertümlichen Losung Carpe diem. In Schnitzlers Erzählung gibt es keine Ausblicke ins Ewige, nur Einblicke ins Zeitliche, das von „hier und jetzt” bedingt wird.

Die lapidare Form dieser Erzählung, der Mangel an Stilwörtern, fast keine Dialoge, denn der Erzähler wiederholt alles in der indirekten Rede, lassen den Eindruck entstehen, dass sie ein psychologisches Studium des Einzelfalls ist. Das Studium soll vielleicht auf die Frage antworten, warum Menschen bestimmte Entscheidungen treffen.

Die Körperlichkeit ist ein brennendes Thema sowohl bei Schnitzler, als auch heutzutage in der feministischen Literatur. Feministinnen werfen oft vor, dass der Frauenkörper in der Männerkultur zum bloßen Gegenstand gemacht wurde. Die gescheiterte Braut aus Schnitzlers Erzählung ist aber ihrer Körperlichkeit auf eine kluge Weise bewusst. Sie benutzt bewusst ihre Macht, die sie über den Männern hat. Die Frau ist hier kein sexuelles Objekt. Sie ist ein sexuelles Subjekt. Was anderes wird hier geäußert, wenn nicht die Macht des Geschlechtes?

Die Teilung in Frauentypen ist ein gewisses Stereotyp, mit dem eine der berühmten Feministinnen, Simone De Beauvoir gekämpft hat. Sie präsentierte die These, dass die Unterdrückung der Frau gesellschaftlich bedingt sei: „Man wird nicht als Frau geboren, man wird es.” Ihrer Meinung nach existiert keine irgendwie geartete Essenz der Frau. Frauen wurden von den

\footnotetext{
${ }^{8}$ A. Schwarzer, Simone de Beauvoir, Rorohlt Taschenbuch Verlag, Hamburg 2007, S. 161.
} 
Männern zum „Anderen Geschlecht” gemacht. In dem philosophischen Sinn bedeutet das, dass „sich der Mann als das Absolute, das Essentielle, das Subjekt setzt, während der Frau die Rolle der Anderen, des Objekts zugewiesen wird." Frauen werden immer in einer Relation zu Männern definiert. Sie wurden von dieser Abhängigkeit gefesselt. De Beauvoir meint, dass Frauen sich mit einer passiven Rolle begnügen müssen, wenn sie ihrer „Weiblichkeit” gerecht werden wollen.

Einen oppositionellen Standpunkt präsentierte Otto Weininger, ein Psychologe und Philosoph, der zu Beginn des 20. Jahrhunderts schaffte. ${ }^{10}$ Seiner Meinung nach empfängt die Frau das Leben erst durch den Mann „aus zweiter Hand". Das bedeutet, dass Frauen überhaupt nicht selbstständig sind, um ihr eigenes Leben autonom zu führen. Dessen sind Frauen selbst schuldig. Ihre Schuld liegt an ihrem Wesen. Die Männer zeigen den Weibern die echte große Welt nur aus Edelmut, weil die Werte des höheren Lebens der Frau ebenso unzugänglich wie die Welt der Ideen sind. Wie Weininger schreibt: „Die Frau ist nur sexuell, der Mann ist auch sexuell.” Mit diesen Worten wird die Frau zum Objekt gemacht.

Die beiden oben genannten Sichtpunkte sind in Schnitzlers Erzählungen präsent. Einerseits zeigt er, dass Frauen oft als Objekte von Männern betrachtet werden, was sie zu Opfern des männlichen Egoismus macht, andererseits stellt Schnitzler sehr bildhaft dar, wie stark sich Frauen bemühen, sich von diesen Fesseln zu befreien.

\section{Fräulein Else}

Eins der besten Beispiele des psychologischen Models eines unschuldigen Opfers gab Schnitzler in der Erzählung Fräulein Else an. Das Werk stellt eine gelungene Probe der Psychoanalyse der Protagonistin dar. Ihre Zerrissenheit wird in Form eines inneren Monologs wiedergegeben.

Die neunzehnjährige Protagonistin Else verbringt mit ihrer Tante und ihrem Vetter Ferien in Italien. Eines Tages bekommt sie einen Brief von ihrer Mutter, in dem steht, dass ihre Familie Schulden hat. Else muss dringend das

\footnotetext{
${ }^{9}$ S. z.B.: S. de Beauvoir, Das andere Geschlecht, Übers. E. Rechel-Mertens, Fritz Montfort Rowohlt Verlag, Hamburg 1951.

${ }^{10}$ O. Weininger, Geschlecht und Charakter, 19. Aufl., Wien-Leipzig 1920.
} 
Geld von einem Familienfreund dem Herrn von Dorsday leihen, der zufällig in demselben Hotel wie Else eine Unterkunft fand. Herr von Dorsday geht darauf ein, aber unter einer Bedingung: Else soll sich ihm nackt zeigen!

In Elses Gedanken liest man wie in einem Buch. Der Bewusstseinsstrom spielt in dieser Erzählung eine sehr wichtige Rolle: Der Leser wird davon überzeugt, dass er es mit einem neunzehnjährigen Mädchen zu tun hat. Ihre Gedanken fließen chaotisch, konzentrieren sich gleichzeitig auf viele Sachen, sind ungeordnet und entdecken Interessen, die typisch für ein junges Mädchen der Jahrhundertwende sind: Musik, Aussehen, Gerüchte, die Vorbereitung zur Rolle einer Ehefrau. Das Fräulein kann man mit den Wörter: Unschuld und Sorglosigkeit charakterisieren. Else ist in ihrem Weltbild keusch und naiv. Sie ist immer noch ein Kind. Sie verhält sich manchmal komisch, wenn sie sich zum Beispiel fragt: „Trinkt man oder raucht man Haschisch?”11 Darauf, dass sie zum Teil immer noch ein kleines Mädchen ist, weist ihr naives Denken über eine Villa an der Riviera hin. Das Leben, das sie sich ausmalt, ist wie eine Idylle.

Im Unterbewusstsein spürt sie aber, dass diese Idylle bald unterbrochen wird. Sie weiß, dass sie einen Expressbrief von ihrer Mutter bekommen hat. Sie will ihn nicht lesen, weil sie denkt, dass im Expressbrief nur etwas Unangenehmes stehen muss. Aber das Schlimmste, was sie sich vorstellen kann, ist eine Nachricht, dass sie nach Hause zurückkehren soll. Nach einer Weile wenden sich aber ihre Gedanken auf ihr Aussehen und sie widmet ihre Aufmerksamkeit der Kleidung. Plötzlich denkt sie darüber nach, ob die Mutter ihren Vater betrogen hat. In diesem Gedankenstrom sieht man, dass sie emotionell unreif ist. Sie versetzt sich schnell in Exaltation. Elses Gefühlzustand ist unstabil und lässt sich nicht einfach kontrollieren. Das ist aber typisch für ein Mädchen, das erst in das Leben von den Erwachsenen eintritt.

Von den Interpretern des Werkes Schnitzlers wird Else als lebenslustiges Geschöpf beschrieben, das von einer Reihe erotischer Episode zu berichten weiß. ${ }^{12}$ Was aber ein von den Interpretern, William H. Rey, ihre „Empfänglichkeit für die Schönheit der Welt" nennt, kann es eher für eine Exaltation gehalten werden. Rey meint, dass Else „ein echtes Verhältnis zur äußeren, wie zur inneren Natur [hat]"13. Wahrscheinlich versteht er unter den Worten

\footnotetext{
${ }^{11}$ A. Schnitzler, Leutnant Gustl und andere Meistererzählungen, Langen Müller, München 2002, S. 11.

${ }^{12}$ W. H. Rey, Arthur Schnitzler. Die späte Prosa als Gipfel seines Schaffens, Erich Schmidt Verlag, Berlin 1968, S. 51.

${ }^{13}$ Ebd.
} 
„echtes Verhältnis” einfach „Ehrlichkeit”, weil die Gefühle, die aus der ErstPerson-Perspektive geäußert werden, immer wahr sind, wenn diese Person davon überzeugt ist. Die Frage ist aber, ob Else tatsächlich bei der Äußerung ihrer Gefühle ehrlich ist? Es ist klar, dass Elses Gefühle am Anfang der Erzählung eher als künstlich dargestellt werden. Man spürt etwas Unnatürliches in ihnen. Sie reagiert mit bestimmten Gefühlen, wie zum Beispiel Begeisterung, nur deswegen, weil sie diese Reaktion einfach passend findet. So passiert es zum Beispiel, dass wenn sie eine Gebirgslandschaft sieht, sie denkt, sie müsse sie bewundern. Else hat noch keine feste Persönlichkeit. Deswegen spielt sie eine Rolle, die sie mit der Zeit entweder assimilieren oder ablehnen und durch eine andere Haltung ersetzen wird. Sie prüft die Verhaltensweisen in verschiedenen Situationen. Sie ist exaltiert, weil sie dabei übertreibt.

Mit den richtig echten Gefühlen von Else hat man erst dann zu tun, wenn sie ein Angebot von Dorsay bekommt. Danach enthüllt sie sich mental. Vorher war das nur ein Schauspiel. Deswegen wundert es, wenn man bei Rey liest, dass sie Zugang zu allen wesentlichen Bereichen des Menschlichen hat. Dazu gehört zunächst die Sphäre des Natürlichen, Sinnlichen, Triebhaften. ${ }^{14}$ Aber der Mensch hat keinen Zugang zu den eigenen Trieben. Was sich einfach beweisen lässt, ist eben die These, dass Else am Anfang keine echten, sondern künstliche, ausgedachte Gefühle zeigt. Man kann es auf diesem Grund ausschließen, dass sie an Nacktheit als an etwas Schmutziges denkt. Vorher hat es sie nicht gestört, sich in ihren Vorstellungen nackt auf dem Marmor zu sehen. Nach Dorsays Angebot ist sie von der Idee empört. Sie wurde beleidigt. „Ich bin ja ein junges Mädchen. Bin ein anständiges junges Mädchen aus guter Familie. Bin ja keine Dirne..." ${ }^{15}$ Aber zum Teil stimmt Rey der These über die späte Entdeckung ihrer wahren Gefühle zu, indem er schreibt, dass ihr Narzissmus erst eintritt, nachdem es sich herausgestellt hat, dass sie einsam und hilflos ist, also nach dem Angebot von Dorsay. ${ }^{16}$

Bemerkenswert ist in Fräulein Else auch die Einstellung des Mädchens gegenüber dem eigenen Körper. Sie hat ein spezifisches Vertrauen zu sich selbst, das durch eine Entwicklung des Körperbewusstseins entsteht. Else spielt mit ihrer Erotik, aber in einer gewissen Verbergung. Sie beobachtet ihren Körper, wenn sie allein im Zimmer ist. Rey bezeichnet ihr Verhalten sogar als nicht

\footnotetext{
${ }^{14}$ Ebd.

${ }^{15}$ A. Schnitzler, Leutnant Gustl und andere Meistererzählungen, S. 72.

${ }^{16}$ Vgl. W. H. Rey, Arthur Schnitzler, S. 51.
} 
normal. ${ }^{17}$ Er meint die Szene, wenn Else ihr Spiegelbild beobachtet und zu sich selbst spricht: „Ach kommen Sie doch näher, schönes Fräulein. Ich will Ihre blutroten Lippen küssen. Ich will Ihre Brüste an meine Brüste pressen." ${ }^{18}$ Die Szene wird aber durch Elses Leid gerechtfertigt. Rey schreibt: „Das Pathologische der Szene ist überschattet von der Tragik Elses, die kein echtes Du finden, kein echtes Gespräch führen kann."19

Ganz im Gegenteil, ist Else eine gute Beobachterin ihres Körpers. Sie kann auch ihren psychischen Zustand mit bestimmten Körperreaktionen verbinden: „[...] die vorige Nacht habe ich so miserabel geschlafen. Freilich, es sind gerade diese Tage. Drum hab' ich auch das Ziehen in den Beinen." ${ }^{20}$ „Diese Tage" bedeuten die Periode. Else meint, dass sie deswegen extreme Stimmungen von der Depression zur Begeisterung hin hat. Das ist sehr schwer für einen Mann das zu verstehen - das ist der Aspekt der Frauenpsyche, der nur für Frauen reserviert ist. Nur die Frauen können sich in diesen bestimmten Zustand einfühlen. Doch gibt Schnitzler einen gelungenes Abbild der Situation wieder.

Körperlich reagiert Else in der Situation der höchsten Spannung, wenn sie offen nackt vor Dorsay steht. Nach diesem Akt fällt sie in Ohnmacht. In der ergreifenden Beschreibung wurde die ganze Skala von Emotionen wiedergegeben, die gleichzeitig mit der extremen Körperreaktion verbunden sind. Es hat nicht geholfen, dass Else anfangs die Möglichkeit überlegt hat, sich tatsächlich vor Dorsay zu enthüllen. Zur gleichen Zeit der Überlegungen, zieht sie die Möglichkeit des Selbstmordes in Betracht.

Obwohl Else sich die Möglichkeit überlegt hat, sich nackt vor Dorsay zu zeigen, war das eher eine dramatische Probe, ihr wahres Ich zu überwinden, als eine Strategiebearbeitung. Von der Imagination zu den Taten führt ein sehr langer Weg. Außerdem weiß sie nicht, wie sie reagieren soll. Diese Situation ist für sie völlig neu. Darauf hat sie sich nicht vorbereitet. Und gerade deswegen, weil sie Männer nur als potentielle Ehegatten, Beschützer, aber nicht als eigennützige Zyniker begriffen hat.

Schnitzler entdeckt hier die brutale Wahrheit über die männliche Natur. Und gerade Elses Reaktion auf den Zusammenstoß von ihren Träumen über Männer mit der Wirklichkeit weist auf ihre komplexe, noch wenig entdeckte

\footnotetext{
${ }^{17}$ Vgl. Rey W. H., Arthur Schnitzler, S. 53.

${ }^{18}$ A. Schnitzler, Leutnant Gustl und andere Meistererzählungen, S. 62.

${ }^{19}$ W. H. Rey, Arthur Schnitzler, S. 53.

${ }^{20}$ A. Schnitzler, Leutnant Gustl und andere Meistererzählungen, S. 11.
} 
Persönlichkeit hin. Erst nach dem Schock, den Else erlebte, wird es sichtbar, dass sie viel kompliziertere Erlebnisse als Sorgen um die Kleidung hat. Jetzt ist das kein Spiel mehr. Jetzt geht es um die Moral, um die Rahmen, in denen jedes Individuum sich begreift. Es geht um die Grenze, die jeder Mensch für sich bestimmt. Normalerweise ist das ein langer Entwicklungsprozess, in dem das menschliche Wesen durch Lernen, Sozialisation, Kommunikation mit Anderen Schritt für Schritt verschiedene Modelle seiner Verhaltensweise ausarbeitet. Else hat keine Zeit zum Lernen. Sie wird plötzlich mit einer Situation konfrontiert, die für sie neu ist und in der sie sich nicht zu verhalten weiß. Vielleicht deswegen war der Selbstmord die einzige mögliche Lösung. Sie wurde Opfer ihrer Jugend und ihres Erfahrungsmangels.

Rey interpretiert sehr treffend die Gegenüberstellung von der Frauen- und Männerpersönlichkeit am Beispiel von Else und Dorsay:

Das Paradox der Selbsterfüllung in der Selbsthingabe dagegen tritt uns in manchen Frauengestalten Schnitzlers entgegen. Die männliche Eroberungslust findet ihren Gegenpol in der weiblichen Opferbereitschaft. ${ }^{21}$

Diese Opferbereitschaft wird in Fräulein Else zur Spitze getrieben. Die Hauptprotagonistin begeht Selbstmord. Wie ist es dazu gekommen, dass das psychische Leiden zur Lebensvernichtung geführt hat?

Man muss sich dessen bewusst sein, dass Frauen im 19. Jahrhundert immer noch von Männern abhängig wahren, obwohl sich das Bürgertum im 19. Jahrhundert rasch entwickelte. Dieser Sachverhalt hatte auch einen Einfluss auf die Frauen: Sie konnten sich neue Rollen erlauben, sogar Modedame oder Sportfräulein zu werden. Trotzdem...

...[ist] Fräulein Else das Opfer par excellence, vom Vater praktisch verkauft und im labilen psychischen Zustand einer Männerwelt ausgeliefert, die sie eben erst ein wenig verstehen lernt. Sie ist kein weinerliches, sondern ein sehr selbstbewusstes Opfer. Unter den unendlich vielen weiblichen Opfern in der Literatur ist sie eine, die wir dank des genial geführten inneren Monologs, nicht aus der Entfernung bemitleiden können, auf die wir uns im eigentlichen Sinne einlassen müssen. ${ }^{22}$

\footnotetext{
${ }^{21}$ W. H. Rey, Arthur Schnitzler, S. 17.

${ }^{22}$ R. Klüger, Schnitzlers Damen, Weiber, Mädeln, Frauen, S. 47 ff.
} 
Else stellt sich vor, dass sie eine starke Person ist. In der Tat hat sie aber keine Kraft ihr Schicksal zu beeinflussen. Das einzige, worüber sie verfügt ist ihr Leben. Sie kann entscheiden: Sein oder nicht sein? Das ist die Hauptfrage der Ethik, ob man über den Tod entscheiden darf. Fräulein Else zwingt darüber nachzudenken.

Vielleicht fühlt Else unterbewusst, dass es der einzige Ausgang ist, sich das Leben zu nehmen. Das ist eben etwas Merkwürdiges, wie ein psychischer Zustand dazu führen kann, dass der Mensch nicht mehr existieren will. Es scheint, als ob irgendwelche körperlich-geistliche Kausalitäten bestünden, in denen das Mentale Einfluss auf den Körper hat. Und umgekehrt: Was mit dem Körper passiert, findet eine Abspiegelung in der Psyche wieder. Obwohl mentale Zustände zu der geistigen Sphäre gehören, müssen sie gleichzeitig eine physische Referenz haben. Heutzutage wird das gut von der Neurobiologie bewiesen. Die psychischen Zustände lassen sich empirisch untersuchen und auch durch Medikamente heilen. Ist das nicht eine Ironie, dass Else gerade Veronal einnimmt, aber diesmal nicht um sich zu beruhigen, sondern um für immer einzuschlafen.

Der letzte Teil der Erzählung Fräulein Else schildert also Selbstmord und Tod. Mit dieser Tat flieht Else vor der Welt, in der ihre Unschuld bedroht ist. Eine negative Interpretation des Frauenselbstmordes stellt Weininger dar: „Die Frauen scheuen sich nie, ihr Unglück anderen zu zeigen: weil es eben kein echtes Unglück ist, weil hinter ihm keine Schuld steht, am wenigsten die Schuld des Erdenlebens als der Erbsünde. Der letzte, der absolute Beweis der völligen Nichtigkeit des weiblichen Lebens, seines völligen Mangels an höherem Sein, wird uns aus der Art, wie Frauen den Selbstmord vollziehen. Ihr Selbstmord erfolgt nämlich wohl immer mit dem Gedanken an die anderen Menschen, was diese sich denken, wie diese sie bedauern, wie sie sich grämen oder - sich ärgern werden." ${ }^{23}$

Diese Interpretation lehne ich hier völlig ab. Wenn man mit der Sprache der modernen Psychologie die letzte Entscheidung Elses beschreiben möchte, könnte man sagen, dass Else mit dem Selbstmord das damalige Modell der männlich-weilblichen Beziehungen ablehnt, worin die traditionellen männlichen Erwartungen gegenüber Frauen Hingabe und Gehorsam fordern. „Um ihr Dilemma zu lösen befreit sich [Else] von ihrem Körper, der ein Objekt der ästhetischen, erotischen und ökonomischen Spekulationen ist, und lässt

${ }^{23}$ O. Weininger, Geschlecht und Charakter, S. 378. 
hinter sich nur eine leere Schale, die Dorsay sich jetzt anschauen kann, wie lange er will..."24

Elses Wahl, nicht zu sein, beweist eigentlich ihre Reife und Mut. Um ihrem Schicksal zu entgehen, ein Objekt zu werden, findet Else einen einzigen möglichen Ausweg, der eben ihr Subjektsein bestätigt. Und das ist eben der Selbstmord.

\section{Die griechische Tänzerin}

Nicht nur die Hauptprotagonistin in Fräulein Else, sondern auch andere Gestalten aus Schnitzlers Erzählungen, wie zum Beispiel Die griechische Tänzerin können als Symbole des Leidens gelten. Es scheint, als ob die Opferrolle tatsächlich für Frauen der Jahrhundertwende vom Schicksal geschrieben wurde.

Der Ich-Erzähler, dessen Namen und Profession verborgen bleibt, steht vor einem Haus, wo eine Frau, Mathilde Samodeski, vor einigen Tagen gestorben ist. Er glaubt aber an die Ursache ihres Todes nicht. Wahrscheinlich hatte sie einen Herzinfarkt. Der Erzähler ist aber dessen sicher, dass diese Frau Selbstmord begangen hat. In seiner Erzählung enthüllt er die Gründe dieser Tat.

Psychologisch gesehen stellt die Hauptprotagonistin Mathilde ein gutes Material für Forschungen dar. Erstens ist sie eine unglückliche Ehefrau, die sich viel Mühe gibt, um ihre Frustration zu verbergen. Sie tut so, als ob alles in ihrer Ehe in Ordnung wäre. Schon wieder opfert sich eine Frau in dem Namen höherer Werte. Sie will sich nicht scheiden lassen, aber sie gibt ihrem Mann die Freiheit auf andere Weise zurück. Sie macht aus ihm einen Witwer.

Anders als im Falle des Fräuleins Else haben wir hier mit einem Ehepaar zu tun. Bei einer Idealisierung sollte die Ehe als ein Organismus betrachtet werden. Das Ehepaar bildet eine Seele und einen Körper. Die Ehe ist wie eine Einheit, die aus zwei Individuen besteht. So treffen sich hier die Eigenschaften von den zwei Individuen, die aber in den beiden verkörpert werden können: Liebe und Hass, Treue und Treulosigkeit. Das alles ist eine logisch un-

${ }^{24}$ E. Kuttenberg, Suicide as Performance in Dr. Schnitzler's Prose, [in:] A Companion to the Works of Arthur Schnitzler. Dagmar C. G. Lorenz (Hrsg.), Camden House, NY 2003, S. 335. [Übers. A. P.-Ż.] 
auflösbare Widersprüchlichkeit der Innenwelt dieses Ehepaars. Es gibt doch glückliche Ehepaare, aber diese interessieren Schnitzler nicht. Diese sind... langweilig. Die tiefe Psychologie fängt dort an, wo Anomalität beginnt: „Nur wenige erkennen, dass der höchste Sinn der Freiheit die Bindung ist. Die bindungslosen Abenteurer, die niemals echtes Opfer bringen konnten, bleiben unfruchtbar und verfallen dem Schicksal der Einsamkeit." ${ }^{25}$

Die Bindung ist nicht nur der höchste Sinn der Freiheit, sondern sogar des Lebens. Das aber verstehen eher die Frauen als Männer. Das beste Beispiel ist hier Mathilde. Die sorglose Unwissenheit der Männer ist Glück. Die Frauen leiden dagegen, denn sie sind sich dessen bewusst, dass ihr Leben von Männern abhängt. Mathilde steht vor einem tiefen Existenzproblem, das darin besteht, dass ihr Pflichtgefühl gegenüber ihrem Mann mit dem oft an Selbstzerstörung grenzenden Leiden in Konflikt kommt. Der Ich-Erzähler fühlt sich in die Lage von Mathilde ein, was er mit den folgenden Worten beschreibt:

Und ich sah, wie sie zu ihrem Gatten hinüberschaute und ihm einen Blick zuwarf, der nicht nur eine unendliche Liebe verriet, sondern auch ein unerschüttertes Vertrauen heuchelte, als wäre es wahrhaftig ihre höchste Pflicht ihn im Genuss des Daseins auf keine Weise zu stören. Und er empfing auch diesen Blick - lächelnd, unbeirrt, obwohl er natürlich ebenso gut wusste als ich, dass sie litt und ihr Leben lang gelitten hat wie ein Tier. ${ }^{26}$

Der Ehemann ist grausam, egoistisch, herzlos. Warum also bleibt Mathilde die ganze Zeit über mit ihm? Ist sie heilig oder wahnsinnig? Opferbereit oder masochistisch? Wäre es für diese Ehe nicht gesünder, wenn sie einmal eine große Eifersuchtsszene machen würde? Vielleicht wäre das auch für den Mann endlich etwas reizvolles, was er bisher in anderen Frauen suchte?

Mathilde würde sich aber nie so verhalten, denn sie ist nicht nur Ehefrau, sondern auch Mutter. Sie hat ein kleines Kind und für sie ist das Kind viel wichtiger, als ihre persönlichen Gefühle. Sie verzichtet auf ihren Stolz im Namen der Familie. Sie wird nicht geliebt, aber sie liebt. Obwohl sie finanziell unabhängig ist (es besteht sogar ein Verdacht, dass Gregor sie wegen ihres Geldes geheiratet hat), ist sie in eine unverständliche Relation zu ihrem Mann verwickelt.

${ }^{25}$ W. H. Rey, Arthur Schnitzler, S. 17.

${ }^{26}$ A. Schnitzler, Die griechische Tänzerin, [in:] Erzählende Schriften von Arthur Schnitzler, Bd. I, S. Fischer Verlag, Berlin 1913, S. 317 ff. 
In den Augen ihres Mannes ist Mathilde minderwertig. In der Tat ist sie aber eine Heldin, die die Last eine Ehefrau eines Betrügers zu sein schweigend trägt. Sie ist eine intelligente Frau, die über ein bemerkenswertes Selbstwissen verfügt. Deswegen fällt es schwer zu glauben, dass sie über die Betrügereien ihres Manns nichts weiß. Aber wie lange kann sie diese Situation aushalten? Die Antwort ist hier eindeutig. Kein Mensch und kein Geschöpf, das Gefühle hat, würde das lange ertragen. Was tief in der Seele versteckt bleibt, in den fröhlichen Worten maskiert wird, das findet einen Ausgang in Körpersprache.

Die psychische Konstitution Mathildes findet einen Ausdruck in der unbewussten Körperhaltung, Bewegung, Gestik und Mimik. Ihre Augen sprechen für sie. Nicht ohne Grund nennt man die Augen „Spiegel der Seele'”'[N]ie habe ich die Augen eines weiblichen Wesens so viel Leid ausdrücken - oder verbergen sehen." ${ }^{27}$ - sagt der Erzähler. Die innere psychische Anspannung reagiert Mathilde körperlich ab. Mathilde ist nervös aber nicht neurotisch. Auf eine bestimmte Weise ist sie stark - auch körperlich. Sie kann sich selbst gut beobachten und das unerwünschte Körperverhalten beherrschen.

Mathilde verliert mit ihrer Schlankheit die letzte Chance, ein Objekt der Gier zu sein. Bemerkenswert ist hier ein Kommentar des Erzählers, der doch auch ein Mann ist. Er ist in diesem Falle ein ehrlicher Kritiker, der kalt das Objekt seiner Beobachtung betrachtet, obwohl er an anderen Stellen eine Sympathie für Mathilde zeigt. Er sagt ohne Umschweife, was er sich in einem Fragment über Mathildes Aussehen gedacht hat: „Auch im Theater sprach ich sie einmal, sie war mit ihrer Mutter dort, die eigentlich noch immer schöner ist als sie..."28

Mit diesen Worten stellt er Mathilde im schlechten Licht dar. Sie ist in einer verlorenen Position, weil sie nicht hübsch ist! In diesem Moment lässt sich schon leicht vorhersehen, dass das eins der Gründe ihres Leidens ist. Denn ihr Mann ist als Künstler besonders für Schönheit empfindlich. In dieser Hinsicht weckt Mathilde nicht mehr das Interesse ihres Manns.

Mathilde wählt den Tod. In Schnitzlers Erzählungen kommt dem Tod oft eine ethische Bedeutung zu. In Die griechische Tänzerin entschließt sich die Protagonistin zum Selbstmord und gewinnt in diesem freien Akt ihre letzte Würde. Als vielmals betrogene Frau, lässt sie sich in diesem Akt nicht mehr

27 Ebd. S. 316.

${ }^{28}$ Ebd. S. 305. 
erniedrigen. Sie hat ihr Schicksal in ihrer Hand. Auf diese Weise befreit sie sich, um nichts mehr zu fühlen. Alles stirbt mit ihr.

In den erzählenden Werken von Schnitzler spielt der Tod eine wichtige Rolle. Der Tod ist schrecklich, grausam und sinnlos, aber gleichzeitig, weil er das absolute Nichts bedeutet, ist er der einzige Ausweg, die einzige Möglichkeit der Wahl. Mathilde entscheidet sich für das Nichtsein.

Ihr Tod ist wie ihr Leben. Das ist ein Betrug. Wie gesagt, die offizielle Ursache Mathildes Todes ist Herzinfarkt. Sogar damit will sie ihren Mann schützen. Die gesellschaftliche Meinung über ihn wäre viel negativer, wenn es ans Licht käme, dass seine Frau Selbstmord begangen hat. Auch in Die griechische Tänzerin fällt eine Frau einem Mann zum Opfer - diesmal sogar ihrem Ehegatten. Das ist das Dramatische in der ganzen Geschichte, dass gerade der Ehemann, der Haushüter sein sollte, zum Henker seiner Ehefrau geworden ist.

\section{Kommentar}

Die Psychologie der Jahrhundertwende wurde von der Theorie der Psychoanalyse Sigmund Freuds dominiert. Obwohl Schnitzler Freud nie persönlich getroffen hat, haben sie miteinander Briefe gewechselt. Bestimmt lernte Schnitzler von Freud auf diese Weise, wie man die psychischen Geräte analysiert. Für Freud sind sowohl Männer als auch Frauen in einem Käfig der Kultur geschlossen. Die Kultur ist nach Freud gerade das, was die Menschen beschränkt, aber ihnen gleichzeitig Sicherheit gibt.

Als letzten, gewiss nicht unwichtigsten Charakterzug einer Kultur haben wir zu würdigen, in welcher Weise die Beziehungen der Menschen zueinander, die sozialen Beziehungen, geregelt sind, die den Menschen als Nachbarn, als Hilfskraft, als Sexualobjekt eines anderen, als Mitglied einer Familie, eines Staates betreffen.

[...] Schönheit, Reinlichkeit und Ordnung nehmen offenbar eine besondere Stellung unter den Kulturanforderungen ein. Niemand wird behaupten, dass sie ebenso lebenswichtig seien wie die Beherrschung der Naturkräfte und andere Momente, die wir noch kennen lernen sollen, und doch wird niemand gern sie als Nebensächlichkeiten zurückstellen wollen. ${ }^{29}$

${ }^{29}$ S. Freud, Der Mensch als Prothesengott, [in:] Volker Spierling, Lust an der Erkenntnis: Die Philosophie des 20. Jahrhunderts, Pieper Verlag München 1988, S. 321 ff. 
Hinter der allgemeinen Frauencharakteristik stehen die sozialen Funktionen als Mutter und Ehegattin und es steht etwas, was tief in das Bewusstsein geht, etwas Archetypisches, was die letzten Gründe und Zusammenhänge betrifft. Das sind die Attribute, die die Weiblichkeit bezeichnen. Die Frau ist sich ihrer Sexualität bewusst, sie verkörpert Urtriebe und natürliche Neigungen. Die Frau wird hier mit dem Frauenkörper gleichgesetzt. Unbewusst ist hier der Geschlechtstrieb, der die Handlungen motiviert. In dem zweiten Fall steht die Frau im Gegensatz zu allem Irdischen. Sie hat wenig mit dem alltäglichen Leben zu tun. Sie verkörpert Tugend und Würde. Sie ist diejenige, die das Leben in die Welt bringt.

Interessant ist, dass diese Entwicklung der Frauenrolle auch im wirklichen Leben von Schnitzler präsent ist. Es reicht zu erwähnen, dass seine Mutter in vielen Biographien nur als Hausfrau auftritt, während seine Frau, Olga Gussmann, schon einen „wahren” Beruf hat: sie ist Schauspielerin, obwohl es zweifelhaft ist, ob dieser Beruf Hand in Hand mit dem höheren gesellschaftlichen Status geht.

Natürlich gibt's in Schnitzlers Werk Prostitution, Stubenmädel, Näherinnen, auch Gouvernanten und eine gelegentliche Klavierlehrerin, aber das sind alles "Jobs", ausgeübt um ein paar extra Schillinge zu verdienen, es sind keine Berufe. [...] Und doch gab es in Schnitzlers Wien und Schnitzlers Europa schon anspruchsvollere berufstätige Frauen, was auch im Tagebuch gelegentlich registriert wird. ${ }^{30}$

Die realen weiblich-männlichen Beziehungen gibt Schnitzlers Autobiographie "Jugend in Wien" wieder. Hier beschreibt der Autor z.B. eine unglückliche Liebesgeschichte seines Vaters, über die er viele Jahre nach seinem Tod erfuhr.

In der inneren Welt Schnitzlers Erzählungen, die durch entsprechende ästhetische Mittel beschrieben wurden, herrschen Normen und Werte, die Einfluss auf die zwischenmenschlichen Beziehungen der in den Erzählungen auftretenden Gestalten ausüben. Diese Normen und Werte bestimmen die Moral, die aber nicht einmal mit dem Charakter der Protagonisten in Konflikt geriet. Diese Beziehungen, Konflikte, soziale Situationen schaffen ein Modell, gleichzeitig aber bilden sie auch die potentiell realen Verhältnisse. Diese Wiederspiegelung überträgt einen Leser auf die zweite Ebene der

\footnotetext{
${ }^{30}$ R. Klüger, Schnitzlers Damen, Weiber, Mädeln, Frauen, S. 46 ff.
} 
Überlegungen, nämlich in die Wirklichkeit. Hat die Wirklichkeit aus den Zeiten von Arthur Schnitzler in die fiktive Welt des Werkes transzendiert? Wie sah sie aus? Welchen Einfluss hatte sie auf sein Werk?

Die Antwort auf die erste Frage ist positiv. Es ist nie so, dass ein Schriftsteller getrennt von seiner Epoche schafft. Das ist einfach ontologisch nicht möglich. Er muss sich natürlich nicht direkt auf die Realität seiner Zeiten beziehen, er muss darüber nicht schreiben, aber er ist notwendigerweise mit ihnen verbunden und geistig-seelisch von ihnen geprägt.

\section{Zusammenfassung}

In jeder Epoche herrscht ein Frauenbild. Frauen mussten sich diesen Vorstellung anpassen, wenn sie Anerkennung in der Gesellschaft erhalten wollten. In Fräulein Else und Die griechische Tänzerin sind Frauen immer in einer Relation zu den Männern definiert. Es scheint, als ob sie von dieser Abhängigkeit gefesselt wären. Das ist das große und tragische Hindernis, das ihnen nicht erlaubt, sich als freies Subjekt durch Aktivität selbst zu entwerfen. Diese Funktion der Frauen formuliert die Philosophin Simone de Beauvoir in der Feststellung, dass Frauen sich mit einer passiven Rolle begnügen müssen, wenn sie ihrer „Weiblichkeit” gerecht werden wollen.

In Die Braut sieht man die Protagonistin, die stark, selbstbewusst und selbstständig ist. Männer stellen hier nur eine Fassade dar. Sie vertritt das Beispiel der Frauen, die nicht mehr als Opfer sondern als reizende Täter beschrieben werden sollen. Schnitzler falsifiziert in diesem Werk die allgemeine Ansicht, die aufgrund bestimmter gesellschaftlicher Anschauungen des 19. Jahrhunderts herrschte, dass Frauen nur sanftmütige, gute, ehrliche, liebe Wesen sind, die sich um Haus und Familie kümmern. So wurde die Frauenrolle wahrgenommen, aber die Wirklichkeit war anders. Dabei ist das Motiv der Körperlichkeit sehr wichtig. Die Frauen benutzen ihr Körperbewusstsein, ihr Selbstwissen, um Macht über die Männern zu gewinnen. Was anderes ist hier geäußert, wenn nicht die Macht des Geschlechtes? Die Frau ist hier nicht ein sexuelles Objekt, sondern auch ein Subjekt, welches die Männer manipuliert.

Einerseits zeigt Schnitzler, dass Frauen oft nur als Objekte von den Männern wahrgenommen werden, was sie zum Opfer des männlichen Egoismus macht, andererseits stellt Schnitzler sehr bildhaft dar, wie stark sich die Frauen bemühen, sich von diesen Fesseln zu befreien. Das machen sie nicht ein- 
mal skrupellos. Das zweite Bild stellt also eine eigennützige, grausame Frau dar, die Männer instrumental betrachtet. Ihre hemmungslose, opportunistische Verhaltensweise vernichtet ihren Partner.

Schnitzler beurteilt nie diese Frauengestalten. Er erzählt bloß ihre Geschichten. Er sagte oft, dass ihn nicht das Individuum, sondern der Fall interessiert. Er untersucht diese Fälle wie ein Wissenschaftler und beschreibt sie auf eine ausgezeichnete literarische Weise. Obwohl er selbst als Mann diese Fälle sieht, schreibt er eher für Frauen als für Männer. Denn die Frauen können am besten verstehen, wie treffend seine Überlegungen von der weiblichen Natur sind.

Die Frauenbilder in Schnitzlers Erzählungen und Theaterstücken sind so unterschiedlich, aber dadurch glaubwürdig, dass sie sogar nach Schnitzlers Tode am 21. Oktober 1931 nicht vergessen wurden. Vor allem muss man aber sagen, dass diese Bilder auch heutzutage aktuell sind, was z.B. die Verfilmung der „Traumnovelle” von Stanley Kubrick im Jahre 1999 zeigt. $^{31}$

\section{Literaturverzeichnis}

Primärliteratur

Schnitzler A., Fräulein Else und andere Erzählungen, Fisher Taschenbuch Verlag, Frankfurt am Main 2006.

Schnitzler A., Leutnant Gustl und andere Meistererzählungen, Langen Müller, München 2002.

Schnitzler A., Die griechische Tänzerin. In: Erzählende Schriften von Arthur Schnitzler, Bd. I, S. Fischer Verlag, Berlin 1913.

Schnitzler A., Die Braut, [in:] Gesammelte Werke. Die erzählenden Schriften, 2 Bände, Bd. 1, Frankfurt am Main 1961.

Sekundärliteratur

Freud S., Der Mensch als Prothesengott, [in:] Volker Spierling, Lust an der Erkenntnis: Die Philosophie des 20. Jahrhunderts, Pieper Verlag, München 1988.

Freud S., Vorlesungen zur Einführung in die Psychoanalyse, Bd. I, 2. Aufl., Frankfurt am Main 1970.

Freud F., Totem und Tabu: Einige Übereinstimmungen im Seelenleben der Wilden und der Neurotiker, Fischer Bücherei, Frankfurt am Main 1956.

\footnotetext{
${ }^{31}$ Stanley Kubrick (Regie), Eyes wide shut, 1999.
} 
Freud S., Das Unbehagen in der Kultur, [in:] Das Unbehagen in der Kultur und andere kulturtheoretische Schriften. Psychologie Fischer, Frankfurt am Main 2001.

Jung C. G., Die Archetypen und das kollektive Unbewusste, 8. Aufl., Walter, Olten 1992. Jung C. G., Der Mensch und seine Symbole, übers. Klaus Thiele-Dohrmann, 17. Aufl., Patmos, Düsseldorf 2009.

Klüger R., Schnitzlers Damen, Weiber, Mädeln, Frauen [Vortrag vom 25. Mai 2000], Picus Verlag, Wien 2001.

Lorenz D. G. C. (Hrsg.), A Companion to the Works of Arthur Schnitzler, Camden House, New York 2003.

Rey W. H., Arthur Schnitzler. Die späte Prosa als Gipfel seines Schaffens, Erich Schmidt Verlag, Berlin 1968.

Scheible H., Literarischer Jugendstil in Wien, Artemis Verlag, München und Zürich 1984.

Scheible H., Liebe und Liberalismus. Über Arthur Schnitzler, Aisthesis Verlag, Bielefeld 1996.

Schopenhauer A., Parerga und Paralipomena: Über die Weiber, SW, Bd. 5, Suhrkamp, Frankfurt am Main 1989.

Schwarzer A., Simone de Beauvoir, Rorohlt Taschenbuch Verlag, Hamburg 2007.

Tarnowski-Seidel H., Arthur Schnitzler: Flucht in die Finsternis. Eine produktionsästhetische Untersuchung, 2. Aufl., Wilhelm Fink Verlag, München 1991.

Urbach R., Schnitzler-Kommentar zu den erzählenden Schriften und dramatischen Werken, Winkler Verlag, München 1974.

Weininger O., Geschlecht und Charakter, 19. Aufl., Wien-Leipzig 1920.

\section{Abstract \\ Literary Psychoanalysis of Women's Nature in the Stories of Arthur Schnitzler}

The aim of this article is an outline of the images of women who masterfully portrays Schnitzler in his works. The analysis is provided on the examples of Die Braut, Fräulein Else and Die griechische Tänzerin and refers to the psychological and philosophical foundations of mind-body problem. On the one hand, it refers to the psyche and soma and on the other hand to society and the male-dominated culture as determinants of women existence.

Key words: women, psychoanalysis, nature, existence, drives, Freud, Schnitzler 